Anaesthesist 2011 · 60:605-606 DOI 10.1007/s00101-011-1923-y

Online publiziert: 20. Juli 2011

(c) Springer-Verlag 2011

\author{
G. Huschak ${ }^{1}$ U.X. Kaisers ${ }^{1,2}$ \\ ${ }^{1}$ Klinik und Poliklinik für Anästhesiologie und Intensivtherapie, \\ Universitätsklinikum Leipzig \\ ${ }^{2}$ IFB AdipositasErkrankungen, Universitätsmedizin Leipzig
}

\title{
Anästhesie bei bariatrischer Chirurgie
}

\section{Komorbidität determiniert die Ergebnisqualität}

Infolge der Adipositasepidemie in den entwickelten Ländern der Welt [1] hat die bariatrische Chirurgie in jüngerer Zeit einen erheblichen Aufschwung genommen. Bereits in den 1950er und 1960er Jahren waren erste intestinale BypassOperationen (ilealer, jejunoilealer oder Dünndarm-Bypass) durchgeführt worden. Diese zielten primär auf die operative Behandlung von Magenulzera und Tumoren ab. Dabei kam es ungewollt auch zu Magenverkleinerungen oder Bypässen, sodass die Patienten nach diesen Operationen relevante Gewichtsverluste erlitten. Aufgrund erheblicher Nebenwirkungen wie Mangelernährung, bakterielle Überwucherung und Lebererkrankun- gen [2] wurde die Sinnhaftigkeit des jejunalen Bypasses zur Gewichtsreduktion allerdings grundsätzlich in Zweifel gezogen. Dies führte zur Entwicklung des MagenBypasses durch Mason u. Ito [3].

Bei der Behandlung der Adipositas scheint es mittlerweile klar zu sein, dass ein nachhaltiger Gewichtsverlust in der Mehrzahl der Fälle von morbider Adipositas nur durch eine operative bariatrische Intervention erreichbar ist. $\mathrm{Ob}$ die bariatrische Chirurgie allerdings auch das Langzeitüberleben von morbid-adipösen Patienten verbessern kann, ist derzeit unklar. Nach einer aktuellen retrospektiven Arbeit von Maciejewski et al. [4] profitieren insbesondere jüngere Frauen von der bariatrischen Chirurgie, während für ältere Männer (49,6 $\pm 8,3$ Jahre) kein verbessertes Langzeitüberleben während einer „Follow-up“-Erhebung von im Mittel 6,7 Jahren nach Adipositaschirurgie gezeigt werden konnte. Wie die schwedische SOS-Studie belegt, ist ein Überlebensvorteil jedoch erst nach längeren postoperativen Beobachtungsphasen nachweisbar (Median 13 Jahre; [5]), sodass für eine abschließende Beurteilung ein sehr langer Follow-up-Zeitraum der Patienten notwendig sein wird.

Die Entwicklung minimal-invasiver operativer Techniken, die heute in allen Zentren zur Anwendung kommen [6], führte zu einer deutlichen Verringerung

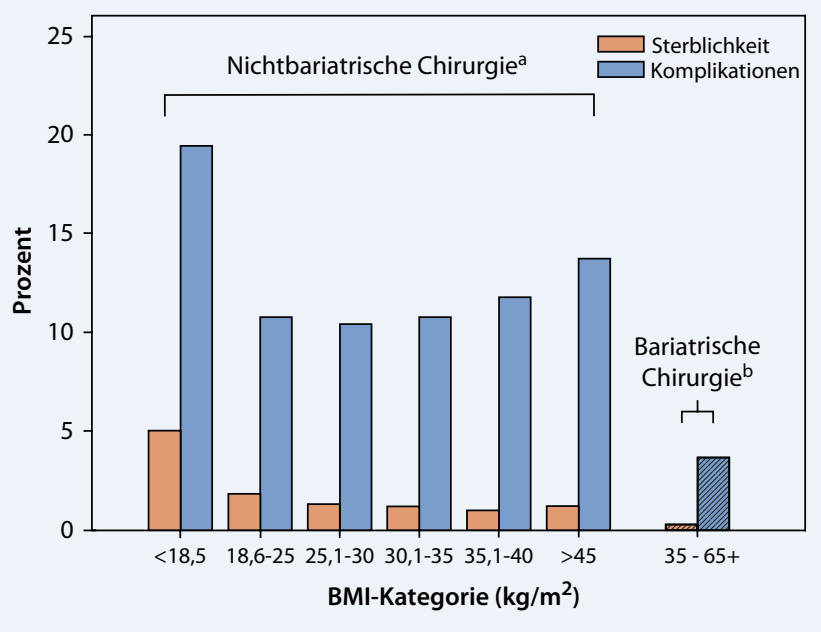

Abb. 1 A 30-Tage-Sterblichkeit und Komplikationsrate bei Patienten mit nichtbariatrischen Eingriffen ( $n=118.707$ Patienten ${ }^{a}$, [9]) und Patienten mit bariatrischen Operationen ( $n=4610^{b}$, [8]). Erstellung nach Daten aus den genannten Quellen. BMI Body-Mass-Index

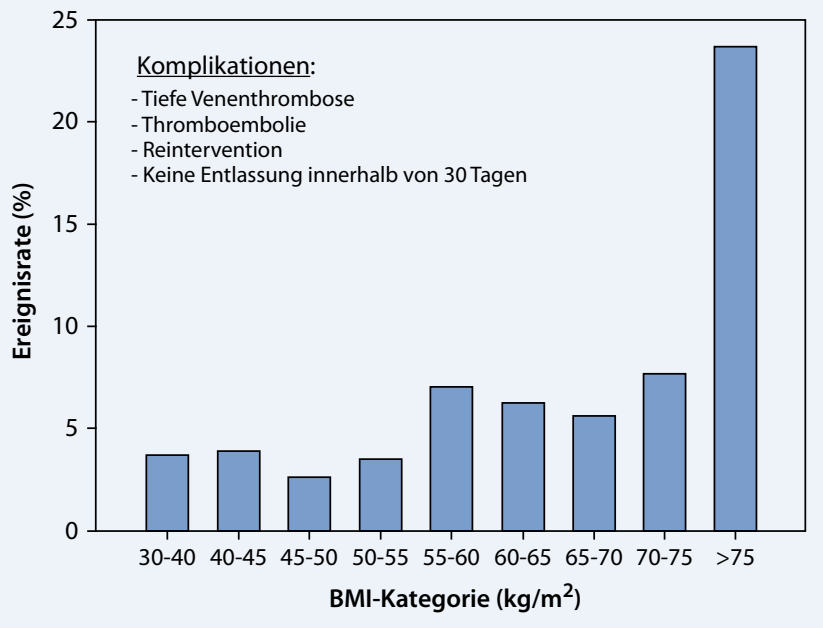

Abb. $2 \Delta$ Perioperative Komplikationen bei Patienten nach bariatrischer Chirurgie $(\mathrm{n}=4607)$ stratifiziert nach BMI-Klassen, BMI Body-Mass-Index. (Daten aus [8]) 
perioperativer, chirurgischer Komplikationen. Diese technisch-operativen Fortschritte und die Erwartungshaltung der Patienten lassen vermuten, dass die Adipositaschirurgie auch in Deutschland ein fester Bestandteil des anästhesiologischen Alltags werden wird. So zählen bariatrische Eingriffe in den USA schon heute zu den am häufigsten durchgeführten viszeralchirurgischen Operationen [7]. Hinsichtlich der präoperativen Vorbereitung und Anästhesie adipöser Patienten gelten grundsätzlich die gleichen pathophysiologischen Überlegungen für bariatrische und auch nichtbariatrische Chirurgie. Das aktuelle Leitthema „Anästhesie bei bariatrischer Chirurgie“ von Konrad et al. dieser Ausgabe von Der Anaesthesist vermittelt dazu praxisrelevantes Fachwissen.

Veränderte Organfunktionen sind für adipöse Patienten aller Altersklassen charakteristisch und können die operative Ergebnisqualität entscheidend beeinflussen. Adipositas ist jedoch per se kein negativer Prognosefaktor für das operative Ergebnis und trotz relevanter Begleiterkrankungen (arterielle Hypertonie in 55\%, obstruktive Schlafapnoe bei $49 \%$, Diabetes mellitus in $33 \%$, Asthma bronchiale in $23 \%$ der Patienten) war in einer aktuellen Längsschnittuntersuchung das Risiko bei bariatrischen Eingriffen vertretbar niedrig (30-Tage-Sterblichkeit im Mittel o,3\%; [8]). Letalität und Komplikationsraten, gruppiert nach Body-Mass-Index(BMI)Klassen, bei Patienten mit nichtbariatrischen und bariatrischen Eingriffen sind in - Abb. 1 dargestellt. Obwohl unterschiedliche Definitionen der perioperativ aufgetretenen Komplikationen verwendet wurden, zeigt sich, dass die elektive bariatrische Chirurgie ein Verfahren mit eher geringem Risiko darstellt. Sofern nach Adipositaschirurgie Komplikationen auftreten, handelt es sich v. a. um tiefe Venenthrombosen, Thromboembolien und die Notwendigkeit operativer Revisionen; diese führen häufig zu einer Verlängerung des Krankenhausaufenthalts. Die genauere Betrachtung dieser Komplikationen ergibt, dass die Ereignisrate mit zunehmendem BMI ansteigt, wobei erst die Gruppe schwerstmorbid Adipöser mit einem BMI $>60 \mathrm{~kg} / \mathrm{m}^{2}$ das hohe Komplikationsniveau kachektischer $\mathrm{Pa}$ tienten aufweist (• Abb. 2). Es erscheint daher sinnvoll, bei der präoperativen Evaluation den Patienten mit Gewichtsextremen besondere Aufmerksamkeit zu widmen.

Nach Risikoadjustierung für die häufigsten Begleiterkrankungen weisen morbid Adipöse bei nichtbariatrischer Chirurgie keine gegenüber Normalgewichtigen erhöhte perioperative Letalität auf [9]. Dies wird unter dem Begriff „Adipositasparadoxon" subsumiert. Es unterstreicht die Bedeutung einer adäquaten perioperativen Diagnostik und Behandlung vorliegender Komorbiditäten für das operative Ergebnis. So tragen untergewichtige Patienten mit einem BMI $<18,5 \mathrm{~kg} / \mathrm{m}^{2}$ das höchste perioperative Risiko. Die anästhesiologische Versorgung adipöser und auch morbid-adipöser Patienten - sei es zu bariatrischer oder nichtbariatrischer Chirurgie - kann demgegenüber bei adäquater präoperativer Vorbereitung sowie zielgerichtetem intra- und postoperativen Management zu vergleichbar guten Ergebnissen führen wie die operative Versorgung Normalgewichtiger [10, 11]. Der Beitrag von Konrad et al. in dieser Ausgabe von Der Anaesthesist präsentiert dazu einen kompakten praktischen Leitfaden.

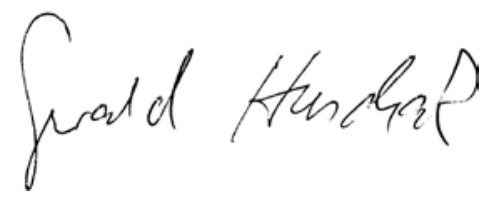

G. Huschak

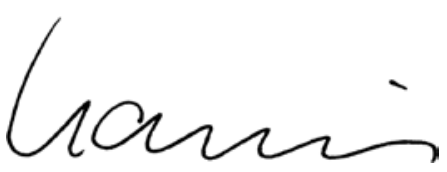

U.X. Kaisers

\section{Korrespondenzadresse \\ Prof. Dr. U.X. Kaisers}

Klinik und Poliklinik für Anästhesiologie und Intensivtherapie, Universitätsklinikum Leipzig Liebigstr. 20, 04103 Leipzig

udo.kaisers@medizin.uni-leipzig.de

Interessenkonflikt. Der korrespondierende Autor gibt an, dass kein Interessenkonflikt besteht.

\section{Literatur}

1. Catenacci VA, Hill JO, Wyatt HR (2009) The obesity epidemic. Clin Chest Med 30:415-444

2. Griffen WO Jr, Bivins BA, Bell RM (1983) The decline and fall of the jejunoileal bypass. Surg Gynecol Obstet 157:301-3

3. Mason EE, Ito C (1967) Gastric bypass in obesity. Surg Clin North Am 47:1345-1351

4. Maciejewski ML, Livingston $\mathrm{EH}$, Smith VA et al (2011) Survival among high-risk patients after bariatric surgery. JAMA 305:2419-2426

5. Sjöström L, Narbro K, Sjöström CD et al (2007) Effects of bariatric surgery on mortality in Swedish obese subjects. N Engl J Med 357:741-752

6. Nguyen NT, Masoomi H, Magno CP et al (2011) Trends in use of bariatric surgery, 2003-2008. J Am Coll Surg, doi:10.1016/j.jamcollsurg.2011.04.030

7. Livingston EH (2010) The incidence of bariatric surgery has plateaued in the U.S. Am J Surg 200:378-385

8. Flum DR, Belle SH, King WC et al; Longitudinal Assessment of Bariatric Surgery (LABS) Consortium (2009) Perioperative safety in the longitudinal assessment of bariatric surgery. N Engl J Med 361:445-454

9. Mullen JT, Moorman DW, Davenport DL (2009) The obesity paradox: body mass index and outcomes in patients undergoing nonbariatric general surgery. Ann Surg 250:166-172

10. Huschak G, Laudi S (2010) Perioperatives Risiko bei Adipositas. Adipositas 4:5-9

11. Kaisers U, Busch T (2010) Perioperatives Management bei Patienten mit Adipositas. Adipositas 4: $1-2$ 